\title{
An Overview of the SCV Simulator for Vehicular Networks Jihene REZGUI ${ }^{1, *}$, Cédryk DOUCET ${ }^{2}$ and Phillipe ALEXANDRE ${ }^{3}$ \\ ${ }^{123}$ Laboratoire Recherche Informatique Maisonneuve (LRIMa), Montreal, Canada \\ *jrezgui@cmaionneuve.qc.ca
}

Keywords: VANET, Active safety, Global perception, Periodic messages, Alert messages, SCV.

\begin{abstract}
The increasing number of vehicles on roads brings more risks associated with vehicular travel. In fact, 50 million victims of accidents with personal injury on the roads are reported around the globe every year. Therefore, it is important to have new technologies that enhance active safety and ensures early detection of dangers. A good solution would be to equip vehicles with wireless, fast and safe communication capacity, called DSRC (Dedicated Short-Range Communication) to coordinate sensory information and perceptions of all vehicles. In order to analyze the success of such technology, we develop a discrete event simulator for modeling vehicle active safety networks, called SCV. Active safety systems are those whose actions begin a few seconds up to a few tenths of a second before the advent of an accident, to prevent or mitigate it. SCV evaluates the success of vehicle-to-vehicle (V2V) and vehicle-to-infrastructure (V2I) communications. It also extends the perceptions of the vehicles environment so that they evaluate situations independently and exploit the redundancy of perception generated maps. Eventually, vehicles react much more quickly in case of danger. Results show that with SCV, we act on cooperative communication systems and we present the overall perception of the road.
\end{abstract}

\section{Introduction}

Intelligent Transportations Systems (ITS) allow the use of emerging technologies, such as wireless communications and sensors to save lives, time and energy. Vehicular ad hoc networks (VANETs) are currently considered a key technology of ITS for bringing more safety on the road. These networks make use of Wireless Access for Vehicular Environment architecture (WAVE) [1-3] to communicate with each other and with the road infrastructure. WAVE defines how vehicles communicate with each other (V2V communications) and with infrastructure equipment (V2I communications) using DSRC; a wireless spectrum allocated by the regulator in the $5.850-5.925 \mathrm{GHz}$ band[4].

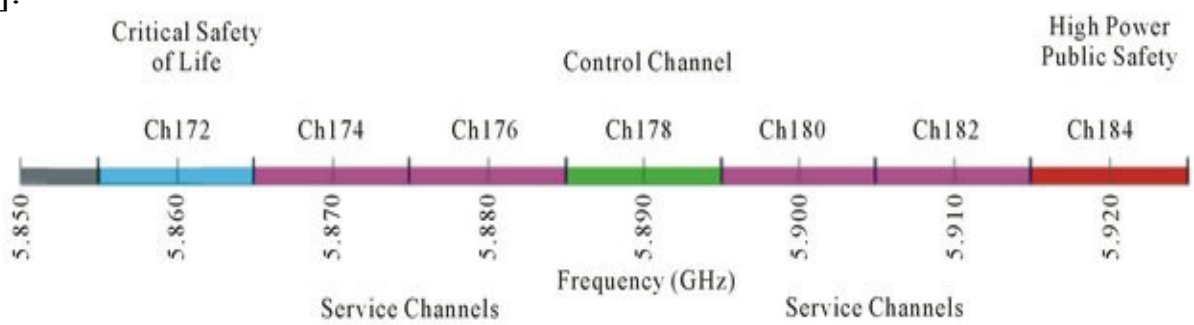

Figure1. DSRC : Wireless spectrum in the $5.850-5.925 \mathrm{GHz}$ band

DSRC spectrum is made up of seven $10 \mathrm{MHz}$ wide channels as shown in Figure 1. Channel 178 is the Control Channel $(\mathrm{CCH})$, which is the default channel for common 
safety communications. The two channels at the ends of the spectrum band are reserved for special uses. The rest are Service Channels $(\mathrm{SCH})$ available for both safety and non-safety use.

VANETs are currently considered an essential technology for future road safety and telematics applications. Different protocols and mechanisms were proposed to achieve better network performance or solve different issues related to the harsh VANET environment. Most of those contributions highlight the importance of the beaconing service where each vehicle periodically broadcasts Cooperative Awareness Messages (CAM [5]) containing collected data about the vehicle's position, speed, etc. This information can be readily available for vehicles in the transmission range. Indeed, some vehicles embed a range of sensors to give them a local perception of their environment (using cameras for example, etc.). The exchange of periodic messages can then enhance their perception by compiling all the information gathered by surrounding vehicles and therefore increase their awareness about upcoming threats (see Figure 8).

It is worth noting the lack of simulation tools that principally focus on vehicular communications and easily offer a simulation platform to various research groups to integrate to their projects based on JAVA programming. Our proposed simulation platform, SCV, is dedicated to presenting global perception [6] on the road based on V2V and V2I communications to avoid accidents. Besides, SCV evaluation aligns with empirical results with more accuracy to reality using Nagakami-m model [10].

In this paper, we introduce a new JAVA based discrete event simulator for modeling vehicular communication networks, called SCV Simulator of Vehicles Communications [11]. The remainder of this paper is organized as follows. Section 2 highlights comparison with other simulation tools. In Section 3, we present Nakagami model while defining its parameters and why we choose it rather than other models. Section 4 presents the design of SCV simulation tool. We finally evaluate the V2V and V2I communications through SCV using DSRC in section 5 and conclude the paper.

\section{Comparison with Other Tools}

This section presents an overview of various commercial and non-commercial network simulation tools [8] in wide use today, and compares them to our proposed simulator SCV.

A. NS-2/NS-3

Presently, NS-2[7] is the most popular network simulator in academic and research groups. Both SCV and OMNET++ present clear separation of simulation kernel and models which is not the case of NS-2. In fact, NS-2 code contains the models alongside their supporting infrastructure, as one indivisible unit [7]. SCV intends to provide a simulation platform for vehicular DSRC communications, on which various research groups can build their own simulation frameworks. Whereas the NS-2 project goal was to build a network simulator. The key difference is the different tools and infrastructure components that SCV can provide that are aimed towards vehicular networking while NS-2 cannot: for instance, GUI-based environment (except for nam in NS), separation of models from experiments, graphical analysis tools, simulation library features and support for hierarchical models.

NS-2 is considered as a dual-language simulator since simulation models are scripts in Tcl (Tool command language), while the simulation kernel and various components (protocols, channels, agents, etc) are implemented in $\mathrm{C}++$ and are made accessible from the Tcl language. In a Tcl script, we define the network topology, the settings 
parameters and the recording statistics. The latter characteristics of NS-2 make the creation of graphical editors difficult to implement. However, it is worth mentioning that NS-3 is an ongoing effort to consolidate all patches and recently developed models into a new version of NS that enhances old versions.

B. OMNET++

OMNeT++ [8] was designed from the beginning to support network simulation on a large scale. In OMNeT++ simulation models are hierarchical, and built from reusable components whenever possible. The proposed simulation software facilitates visualizing and debugging of simulation models in order to reduce debugging time, which traditionally takes up most the simulation time. Its simulation software, as our proposed simulator SCV, is modular, customizable and allows embedding simulations into larger applications such as network planning software.

Both OMNET++ and SCV provide permissions to make digital or hard copies of part or all for personal or classroom use is granted without fees provided that copies are not made or distributed for profit or commercial advantage. Data interfaces are open in both SCV and OMNET++. It is possible to generate and process input and output files with commonly available software tools. Both of them provide an Integrated Development Environment that largely facilitates model development and analyzing results.

SCV offers a more interesting GUI-based execution environment than OMNET++, for instance, the global perception propagation of each vehicle and its environment.

\section{OPNET Modeler}

OPNET Modeler[9] is a commercial product which is freely available worldwide to qualifying universities. OPNET probably has the largest selection of ready-made protocol models (including IPv6, WiMAX, QoS, Ethernet, MPLS, OSPFv3 and many others). OPNET, SCV and OMNET++ provide rich simulation libraries of roughly comparable functionalities. The OPNET simulation library is based on C, while the one in SCV is a Java class library. OPNET's architecture is similar to OMNeT++ and SCV as it allows hierarchical models, but with some restrictions (namely, the "node" level cannot be hierarchical). A significant difference from OMNET++ and SCV is that OPNET models are always of fixed topology, while both OMNeT++'s NED and SCV allow parametric topologies. SCV and OMNeT++ models are simple text files which can be generated e.g. with XML while in OPNET, the preferred way of defining network topology is by using the graphical editor. The editor stores models in a proprietary binary file format, which means in practice that OPNET models are usually difficult to generate by program (it requires writing a $\mathrm{C}$ program that uses an OPNET API).

Both SCV and OPNET provide a graphical debugger and some form of automatic animation which is essential for easy model development. OPNET does not provide source code to the simulation kernel. SCV like OMNeT++, NS-2 and most other non-commercial tools - is fully public-source allowing much easier source level debugging.

OPNET's main advantage over SCV and OMNET++ is definitely its large protocol model library, while its proprietary binary file formats and the lack of source code makes development and problem solving harder. 


\section{Other characteristics of SCV}

- SCV is more of a complete and self-sustaining simulator than a simulation library, because it works as-is, while still maintaining the usability and capabilities of a simulation library.

- Its main advantages over other simulation tools are its graphical representation, its ease of use for simple simulations and its vehicular communications specificity.

- Researchers and developers have a full access to SCV's source code in order to accommodate the simulator to their research goals [11].

\section{Nakagami Model is Implemented in SCV}

\section{A. NAKAGAMI-m in SCV}

To simulate wave propagation in an urban environment, we use the Nakagami-m propagation model [10]. It represents the fluctuation and the variability of a DSRC communication. A radio wave can be victim of interference, reflection or refraction that are too demanding for a simulator to quantify. Hence, it is more appropriate to approximate the propagation of those waves using a statistical model.

There are other propagation models that have been used in commercial and non-commercial network simulation tools. However, SCV implemented Nakagami-m model, because it was shown that it aligns with empirical results with more accuracy than other models. Moreover, it is a more accurate statistical model to reality, because it emphasizes the physical aspect of propagation of radio waves in the air and through obstacles. The value returned by the Nakagami distribution is the received message power. Its formula is expressed as follows:

In which

$$
\begin{array}{l|l}
f(x, m, \Omega)=\frac{2 m^{m} \cdot x^{2 m-1}}{\Gamma(m) \Omega^{m}} \exp \left(-\frac{m x^{2}}{\Omega}\right) & 1 \\
\hline
\end{array}
$$

- $\mathrm{m}$ is a variable which varies according to the distance as follows:

- If distance $<80$ meters: $\mathrm{m}=1.5$, otherwise: $\mathrm{m}=0.75$

- $\Omega$ is the vehicle transmission power.

- $\mathrm{x}$ is a random variable following the Gamma distribution with $\alpha=\mathrm{m} \quad \& \& \beta=\Omega / \mathrm{m}$

In Figure 2, we varied the distance between two vehicles to study the signal strength. This result shows that vehicle power transmission tends to decrease with distance, while retaining a partially random power. This aligns with the observations in reality where the reception power can vary even for two vehicles that are at the same distance from the transmitter. 


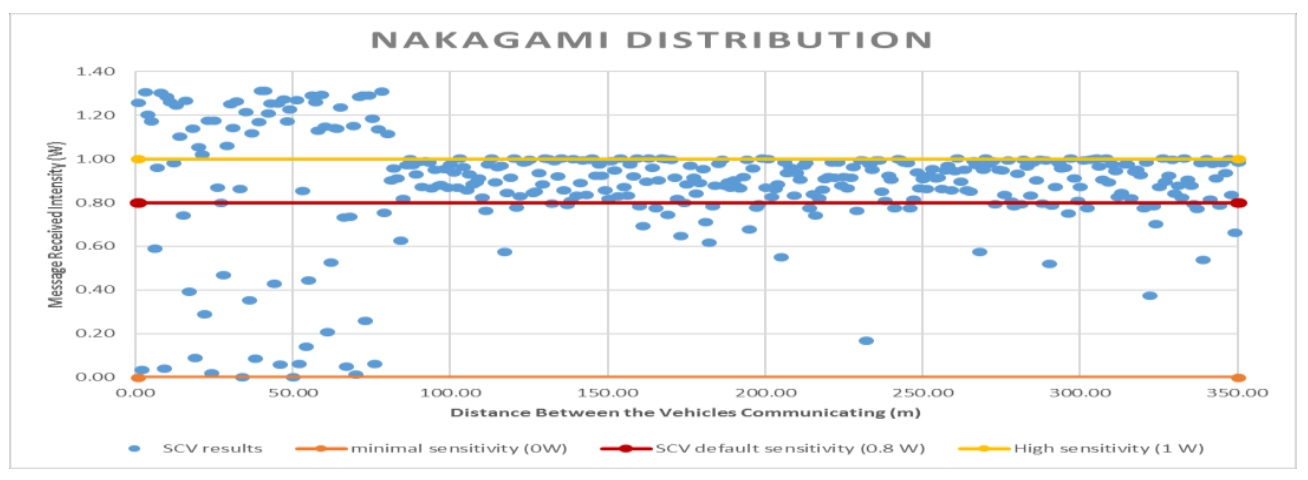

Figure 2. Nakagami-m Model of propagation

B. Why is Nakagami-m fading channel good in practice?

A number of channel models exist to describe the statistics of the amplitude and phase of multipath fading signals. Nakagami-m distribution has the following advantages versus the other models [10]:

- It is a generalized distribution which can model different fading environments.

- It has greater flexibility and accuracy in matching some experimental data than the Rayleigh, lognormal or Rice distributions.

- Rayleigh and one-sided Gaussian distribution are special cases of Nakagami-m model.

Thus, the Nakagami-m channel model is of more general applicability in practical fading channels.

\section{The Design of SCV}

\section{A. SCV Model Structure}

$\mathrm{SCV}$ is a discrete event simulator aimed at simulating vehicular communications. To this end, it was designed to work with SUMO, a reliable traffic simulator. SUMO handles vehicle movements and communicates with SCV so it can simulate network communications between the vehicles. To do so, it uses the TraCI for Java library, which permits communications to the TraCI interface in SUMO (see Figure 3).

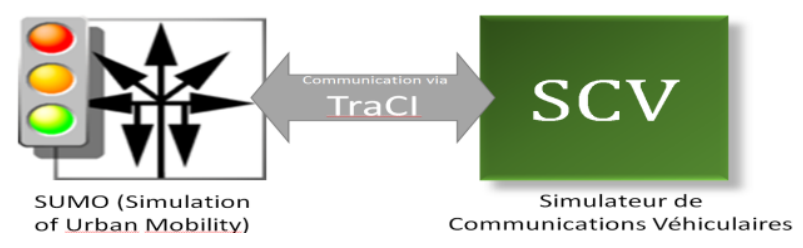

Figure 3. Communications between SUMO and SCV

Also, in order for our simulator to better represent real life, we organized our system (our classes) with the layered network system in mind. We created classes that are associated with different layers of typical network protocols (see Figure 4). Our simulator works as follows, it takes vehicle positions form SUMO, shows corresponding vehicles on the screen and simulates V2V and V2I communications. The 
success of the communications then depends on the wave propagation model (Nakagami-m) and packets collision.

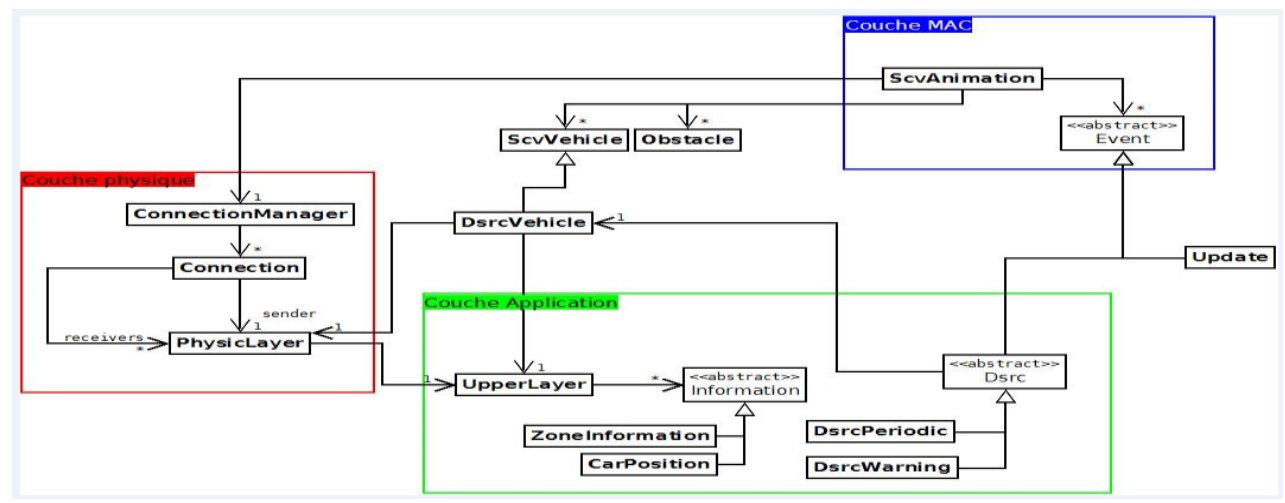

Figure 4. Diagram of classes with UML

\section{B. Input of SCV}

In SCV, you can change different parameters related to the vehicles and their communications. These inputs include:

- Information decay time

- Periodic messages frequency

- DSRC radio sensibility

- Percentage of vehicles with the communication technology

It is also possible to change the vehicle's routes by creating or modifying an existing XML file. This file specifies where vehicles appear and where they are headed on the map. It also dictates the number of vehicles, their maximum speed, their acceleration, their deceleration, etc. (see Figure5).

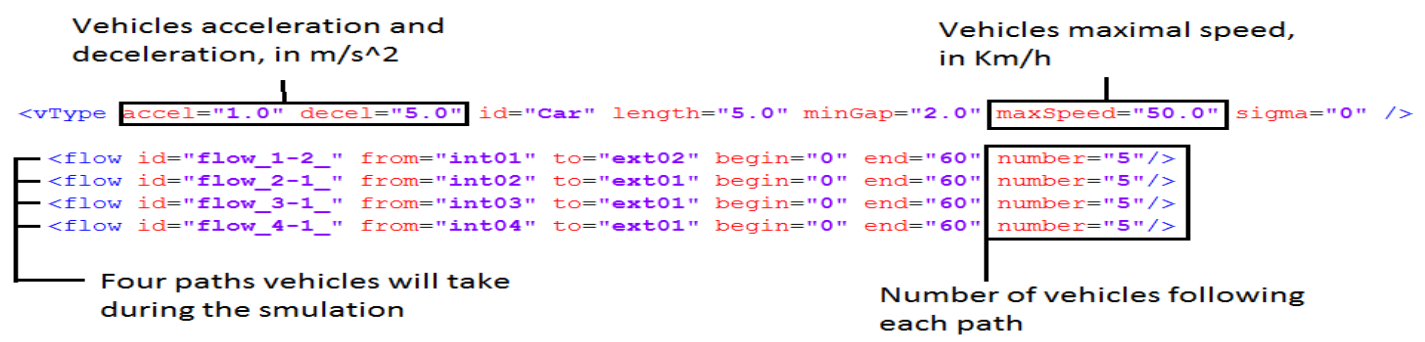

Figure 5. Example of an XML input file

The user can then load their file of choice in SCV and analyze the network scenario in different environments, like in Figure 6.a and Figure 6.b, where two different input files are loaded to show how the behavior of the vehicles can be manipulated.

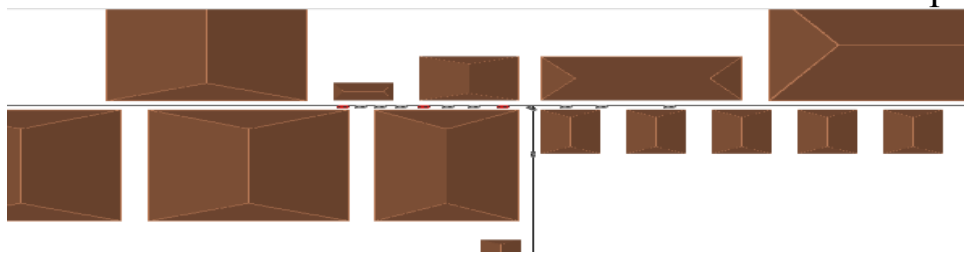

Figure 6.a 


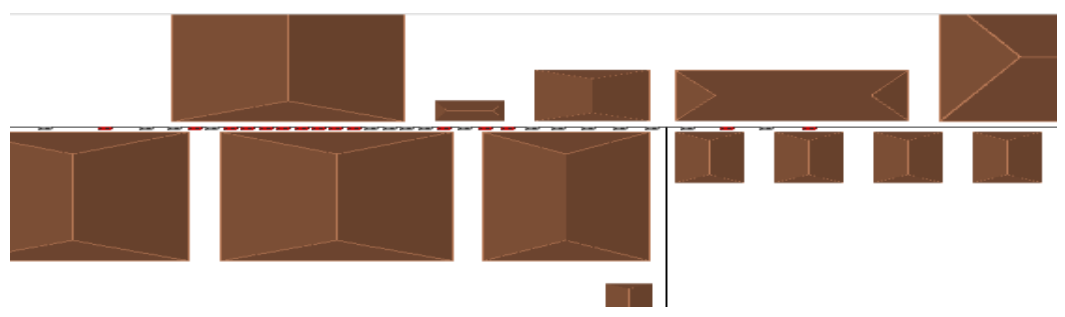

Figure 6.b

Figure 6.a and Figure 6.b are an example of the impact loading 2 different XML input files on the simulation. These two figures were taken at approximately the same simulation time (around 98 seconds)

Another interesting input to note is the crash input. At any given time during the simulation, a user can select a vehicle and choose to make the vehicle crash. The user can then observe the impact on the surroundings and how the message is sent to nearby vehicles.

C. Design of the Simulation Library

The simulation library is designed to be an easy-to-use and modify network simulator, while still being aimed at the simulation of vehicular communications. Consequently, its uses are obviously limited to vehicular simulations. Researchers could still add their own modules to the simulator if they need to see the impact on the spread of information over the vehicular network. Doing so would require knowledge of the Java programming language, but it would still be a basic task due to the low complexity and openness of the simulator (they could change the way the discrete event system works to better suit their needs). In order to follow these goals, SCV was coded as simply as possible, while still being as easy to understand as possible.

D. Contents of the Simulation Library

This section will briefly describe the uses of the different classes as seen in Figure 4.

Firstly, the animation and interactions happen in the ScvAnimation class. It contains the discrete event simulator and all of the vehicles and obstacles present on the map. It then goes through a list of Event and animate the vehicles.

There are currently two main types of Events: Update events and Dsrc events. Update events handle getting the information from SUMO, updating the statistical data for results analysis and passing the new car information to the main animation class. Then, Dsrc events handle the different types of messages exchanged in V2V and V2I communications.

Vehicles that do not have the communication capabilities are represented by the ScvVehicle class and do not contain any networking related objects. Vehicles that use the DSRC technology are called DsrcVehicle and contain an UpperLayer, which handles the analysis of received information, and a PhysicLayer, which handles the receiving and sending of the messages.

These messages are represented by the Information class, which can include car positions and their sensory information. When a message is sent from a vehicle to another, it will send a list of all Information it knows and the receiver will then see if they already know any of these Information and add the ones it does not know to their own list of Information.

Infrastructure is also an important part of the simulation. In fact, one can add antennas (i.e. infrastructure equipment) to the simulation in order to increase the range of communications and to receive V2I messages. 


\section{E. Messages exchanged on SCV}

In our simulator, two types of safety messages are exchanged (see Table 1). The first type is the periodic messages which are sent at regular intervals between vehicles and transmit relevant information to increase the perception and coordination of movements. The second type of messages is the alert (warning) messages which are sent when there is an emergency, such as a collision detection (see Table 2).

This type of message has a higher priority than periodic messages as it is used in an emergency and must be treated as soon as possible. Thus, the exchange of these two message types allows many vehicles to organize and to promote active vehicular safety.

Table1. Different messages exchanged in SCV

\begin{tabular}{|c|c|c|}
\hline Periodic Messages & Safety (periodic + alert) Messages & Alert Messages \\
\hline $\begin{array}{l}\text { Information of the detection zone } \\
\text { - Relate the information collected by } \\
\text { the vehicle sensors, } \\
\text { Example: presence of vehicles in the } \\
\text { blind spot, etc. } \\
\text { - Used to display the access to } \\
\text { Information area }\end{array}$ & $\begin{array}{l}\text { Vehicle location information } \\
\text { - Sent periodically to ensure a } \\
\text { better understanding of the road } \\
\text { - Sent on alert in case of danger }\end{array}$ & $\begin{array}{l}\text { Collision information } \\
\text { - Sent in case of danger (PS) } \\
\text { - Sent if a vehicle testifies an } \\
\text { accident (CF) } \\
\text { - Contains the position of the } \\
\text { vehicles involved in the accident }\end{array}$ \\
\hline $\begin{array}{l}\text { Route information } \\
\text { - Those elements describing the state } \\
\text { of the road } \\
\text { Example: a pothole, a patch of ice, etc. }\end{array}$ & $\begin{array}{l}\text { A pedestrian location information } \\
\text { - Sent periodically to improve the } \\
\text { perception of the neighborhood } \\
\text { - Sent in alerts in case of danger }\end{array}$ & $\begin{array}{l}8 \text { alerting applications to priorities } \\
\text { - Defined by "National Highway } \\
\text { Traffic Safety Administration" in É- } \\
\text { U (see Table 1) }\end{array}$ \\
\hline
\end{tabular}

Table2. Eight safety applications defined by the National Highway Traffic Safety Administration in USA.

\begin{tabular}{|c|c|c|c|c|}
\hline 8 HIGHT-PRIORITY APPLICATIONS & $\underset{\text { Type }}{\text { communications }}$ & $\begin{array}{c}\text { communications } \\
\text { Rate }\end{array}$ & $\begin{array}{l}\text { Threshold } \\
\text { delay }\end{array}$ & $\begin{array}{c}\text { communications } \\
\text { Range }\end{array}$ \\
\hline Traffic Signal Violation: TSV & $12 \mathrm{~V}$ & $10 \mathrm{HZ}$ & $100 \mathrm{~ms}$ & $250 \mathrm{~m}$ \\
\hline Curve Speed Warning: CSW & $12 \mathrm{~V}$ & $1 \mathrm{HZ}$ & $100 \mathrm{~ms}$ & $200 \mathrm{~m}$ \\
\hline Emergency Brake lights: EBL & V2V & $10 \mathrm{HZ}$ & $100 \mathrm{~ms}$ & $200 \mathrm{~m}$ \\
\hline Pre-crash Sensing: PS & $\mathrm{V} 2 \mathrm{~V}$ & $50 \mathrm{HZ}$ & $20 \mathrm{~ms}$ & $50 \mathrm{~m}$ \\
\hline Forward Collision: FC & V2V & $10 \mathrm{HZ}$ & $100 \mathrm{~ms}$ & $150 m$ \\
\hline Left Turn Assist: LTA & $12 \mathrm{~V}$ ou $\mathrm{V} 2 \mathrm{~V}$ & $10 \mathrm{HZ}$ & $100 \mathrm{~ms}$ & $300 \mathrm{~m}$ \\
\hline Lane Change Warning: LCW & V2V & $10 \mathrm{HZ}$ & $100 \mathrm{~ms}$ & $150 \mathrm{~m}$ \\
\hline Stop Sign Assist: SAA & I2V ou V2V & $10 \mathrm{HZ}$ & $100 \mathrm{~ms}$ & $300 \mathrm{~m}$ \\
\hline
\end{tabular}

\section{Results Analysis}

The main purpose of SCV is, of course, to gather data and analyze the impact of certain parameters on vehicular communications. To do this, the simulator can show a vehicle's extended perception by highlighting certain parts of the graphical representation of the simulation. In Figure 7, we can see what a certain vehicle (the green car in the bottom-left corner) can see by highlighting in pink the information it knows. It also knows that a car outside of its line of sight has crashed and that it causes slowdowns in this section of the road.

With this information, it is valuable to say that it is possible to analyze results simply by looking at the vehicles' extended perception. Other results that are available alongside a vehicle's extended perception are its important information, shown by SCV in a panel next to the animation panel. 


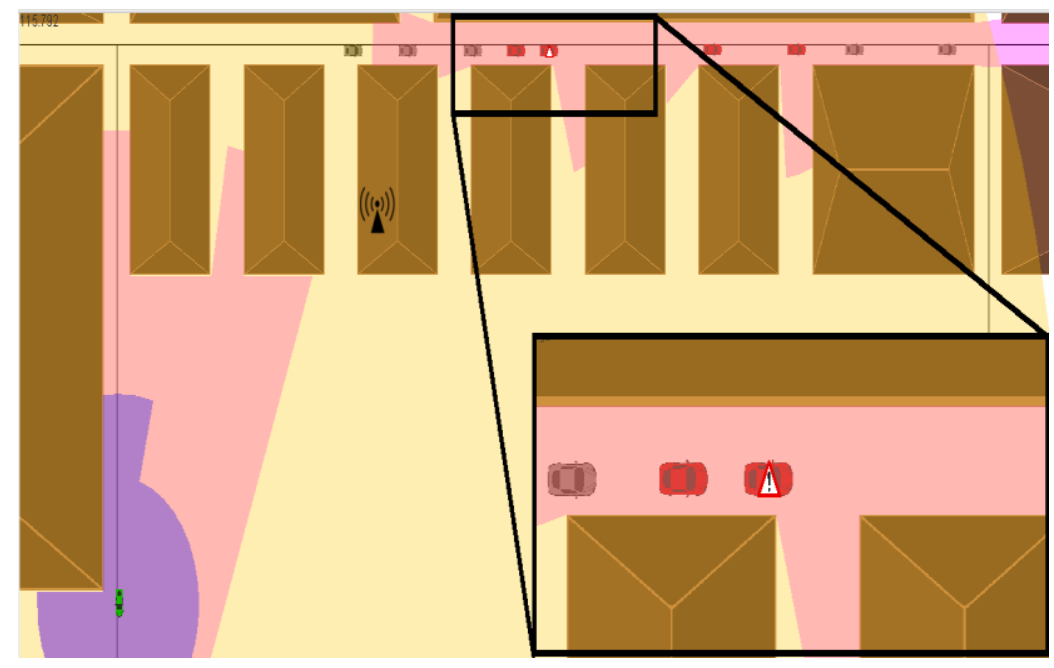

Figure 7. Extended perception with an antenna (infrastructure equipment), in black on a building's roof, helping to increase the communication range

As seen in Figure 8, it is possible to know the selected vehicle's speed, position as well as other relevant data.

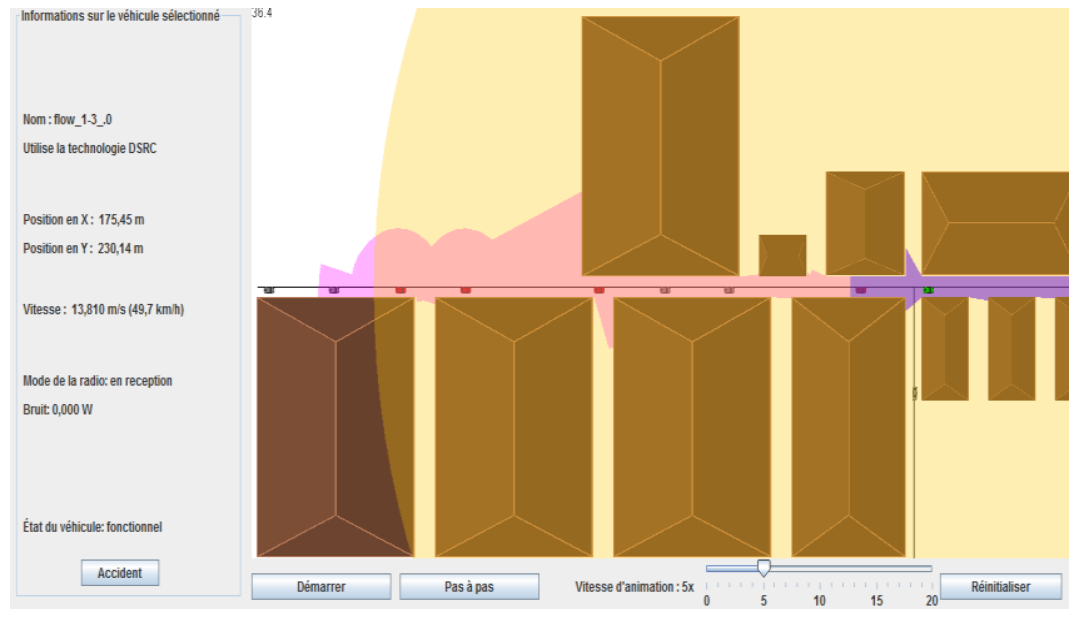

Figure 8. Information about a selected vehicle shown to the left of the screen

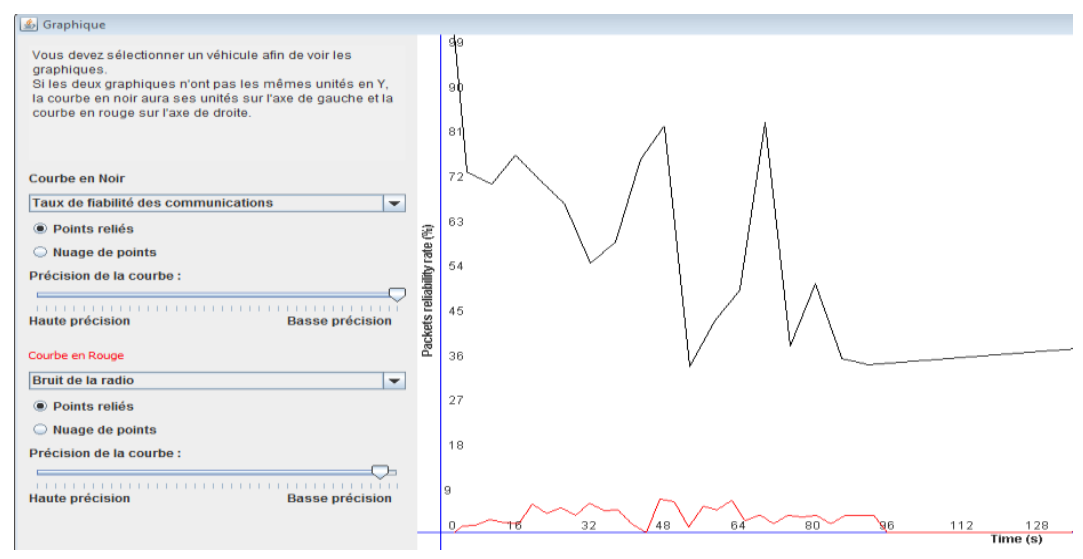

Figure 9. Example of the manipulating of charts in SCV

It is also important to gather actual data in order to produce a chart that is both useful and meaningful. As such, it is important to give researchers a basic set of important data 
and charts to analyze. These include the communication reliability rate, for example. It is also important for researchers to be able to easily add their own charts and analyze them. For this, the chart plotting algorithm has been made with flexibility in mind and will accommodate plenty of type of charts, as seen in Figure 9. It also needs to be able to export the data outside of the application for researchers to manipulate more easily.

An example of the charts in SCV can be seen in Figure 10, where we can see the communication reliability rate of a vehicle in black the number of successfully established connections in red. We can see in this chart that when more messages are successfully received, the reliability rate has the tendency to also rise. This is due to the fact that our vehicle's density stayed mainly similar throughout the simulation, so there was an almost constant amount of tried connections (vehicles with communication capabilities in range).

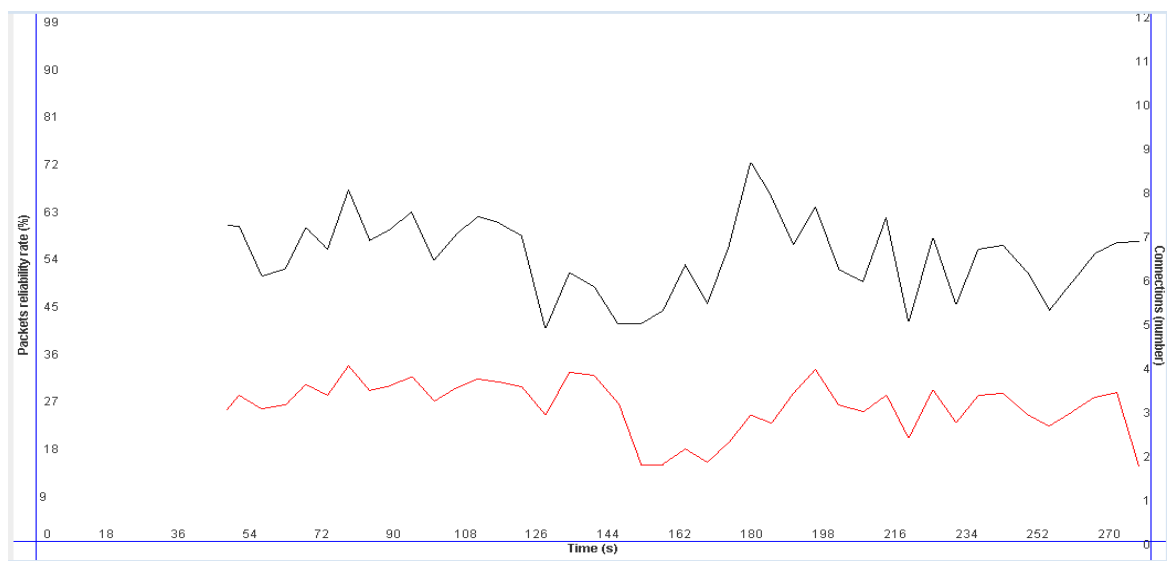

Figure 10. Chart of the Packet reliability rate (top curve in black) and successfully established connections (bottom curve in red). The left vertical axis is for the black curve and the right vertical axis is for the red curve.

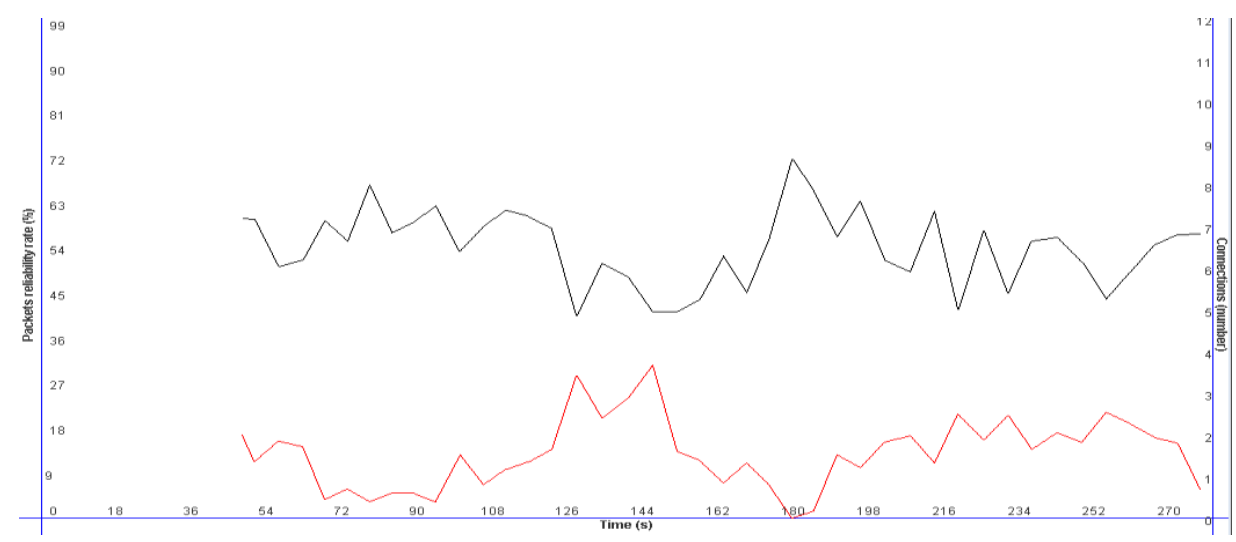

Figure 11. Chart of the Packet reliability rate (top curve in black) and Failed connections by obstacles (bottom curve in red). The left vertical axis is for the black curve and the right vertical axis is for the red curve.

We can also confirm this by changing the successfully established connections chart with the failed connections by obstacles chart, as seen in Figure 11. There, we see that a rise in the failed connections almost consistently lowers the packet reliability rate.

Figure 12 shows simultaneously the successfully established connections and noise power during the exchange of safety messages on DSRC communication. We remark that the noise power impacts the number of successful connections 
Finally, researchers that would like to have more complex charts and values, like the packet reception reliability rate will be able to do so by using SCV's simple chart algorithm.

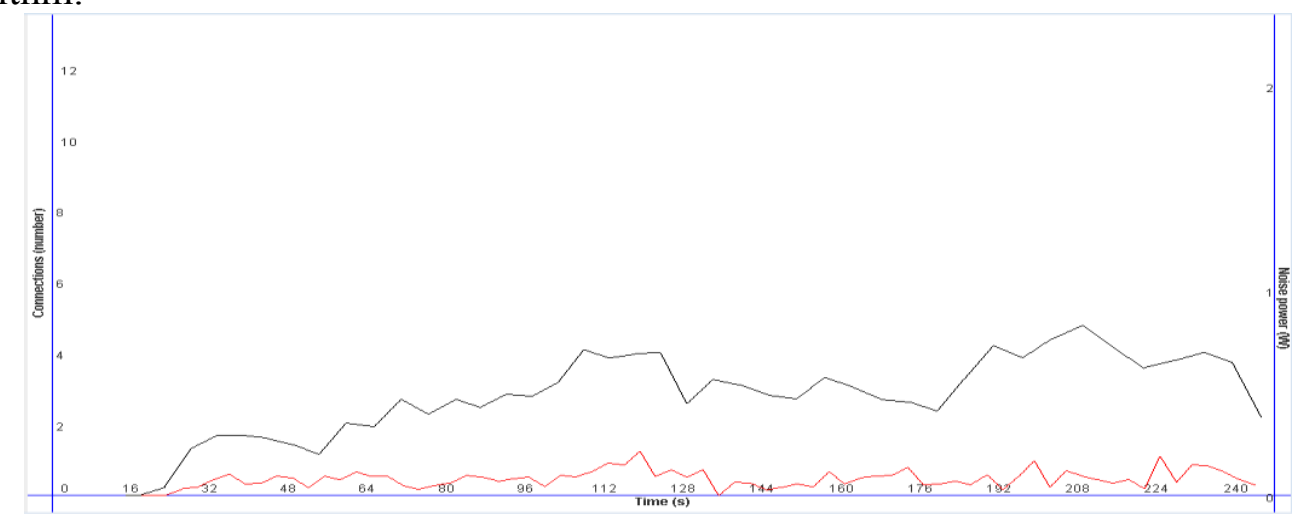

Figure 12. Chart of the successfully established connections (top curve in black) and Noise power (bottom curve in red). The left vertical axis is for the black curve and the right vertical axis is for the red curve.

\section{Conclusion}

In this paper, we show how we build global perception on the road based on V2V and V2I DSRC communication. We develop a new simulator, SCV, which takes into account high-priority safety-oriented periodic or emergency applications to avoid hazardous situations on the roads. Using SCV simulation platform, we showed that the DSRC communications over $802.11 \mathrm{p}$, which integrates Nakagami-fading-m model, enhances the global perception of each vehicle on the road. Besides, through extensive results, we demonstrate that SCV shows good performances in terms of Packet reliability rate in presence of noise or obstacles.

\section{Acknowledgement}

This research was financially supported by the "Fonds Québécois de la recherche sur la nature et les technologies (FRQNT)". We would like to thank Caroline Houle and Nader Chaabouni for their valuable comments.

\section{References}

[1] "IEEE Guide for Wireless Access in Vehicular Environments (WAVE) -Architecture," IEEE Std 1609.0-2013, pp. 1-78, 2014.

[2] J. Rezgui, S. Cherkaoui, O. Chakroun, "Improving delay performance of IEEE $802.11 \mathrm{p}$ vehicular safety communication", published in Int. J. Autonomous and Adaptive Communications Systems 7(1/2): 91-109, 2014.

[3] J. Rezgui, S. Cherkaoui, "About Deterministic and non-Deterministic Vehicular Communications", published in DSRC/802.11p. Wireless Communications and Mobile Computing 14(15): 1435-1449, 2014.

[4] "Trial-Use Standard for Wireless Access in Vehicular Environments (WAVE) Resource Manager," IEEE Std 1609.1-2006, pp. 1-71, 2006. 
[5] E. T. ETSI, "102 638 Technical Report, V1. 1: Intelligent Transport Systems (ITS), Vehicular Communications (VC), Basic Set of Applications, Definitions," ed: June, 2009.

[6] N. Chaabouni, A. Hafid, J. Rezgui, S. Cherkaoui, "Setting up an extended perception in a vehicular network environment: A proof of concept", accepted in IEEE WCNC, 2016.

[7] Bajaj, S., L. Breslau, D. Estrin, K. Fall, S. Floyd, P. Haldar, M. Handley, A. Helmy, J. Heidemann, P. Huang, S. Kumar, S. McCanne, R. Rejaie, P. Sharma, K. Varadhan, Y. $\mathrm{Xu}, \mathrm{H}$. Yu and D. Zappala. 2000. Improving simulation for network research. IEEE Computer. (to appear, a preliminary draft is currently available as USC technical report 99-702)

[8] Varga, A. and Hornig, R. (2008), "An overview of the OMNeT++ simulation environment", Simutools '08: Proceedings of the 1st international conference on Simulation tools and techniques for communications, networks and systems \& workshops: 1--10

[9] OPNET Technologies, Inc. OPNET Modeler. http://www.opnet.com.

[10] N. C. Beaulieu and C. Cheng, "Efficient Nakagami-m fading channel Simulation," in IEEE Transactions on Vehicular Technology, vol. 54, no. 2, pp. 413-424, March 2005. doi: 10.1109/TVT.2004.841555.

[11] Cedryk Doucet, Philippe Alexandre, Jihene Rezgui, "Simulateur de communications véhiculaires", https://gitlab.com/polkio7/SCV, 2016. 